\title{
Near Infrared Interferometry with Large Telescopes
}

\author{
Francesco Paresce \\ European Southern Observatory, Karl-Schwarzchildstr.2, Garching bei \\ Müchen 85748, Germany.
}

\section{Introduction}

First fringes with commissioning instruments and siderostats on both the Keck and VLTI facilities are expected for early 2001.

In this article, we will give a report on the status of the VLTI project as an example of the capabilities of large telescope interferometers and we will describe the strategy for making the VLTI the observatory of the 21st century.

\section{The sub-systems of the VLTI}

The layout of the VLTI is such that a star at infinity illuminates the apertures in the two telescopes with a plane wave that is guided through the Coudé Optical Trains into the Delay Line Tunnel. The delay in arrival of the light at telescope 1 with respect to telescope 2 is compensated by the delay lines so that the beams have zero Optical Path Difference (OPD) when they interfere on the detector in the VLTI laboratory. The field of view of the VLTI is 2 arcsec. However, the dual-feed facility PRIMA will allow picking two stars at the Coudé focus of ATs or UTs each in a 2 arcsec field of view and separated by up to 1 arcmin.

The measurements of the contrast of the fringe pattern and of its phase, i.e. the position of the white light fringe with respect to the nominal zero OPD position are the tasks of the VLT Interferometer. Doing these measurements for many different baselines (different in length and orientation) allows reconstructing an image with the angular resolution of a single telescope with a diameter equal to the longest baseline. Since the longest baselines are $130 \mathrm{~m}$ for the Unit Telescopes and $200 \mathrm{~m}$ for the Auxiliary Telescopes and since the specified precision for the measurements of the fringe contrast is very high, the requirements e.g. for the Delay Line systems are very tough. Here, the beam tilt accuracy has to be better than 1.5 arc seconds ( 15 milli arcsec on the sky) and the absolute position accuracy is $1 \mu \mathrm{m}$ over $65 \mathrm{~m}$ of travel in the tunnel. Similar accuracy values are required for all mirrors and mirror mounts of the VLTI, including the $8 \mathrm{~m}$ primary mirrors. Also, the environmental conditions have to be very stable in order to minimise internal turbulence.

It is the advantage of the VLTI that these considerations have been driving the design of the Unit Telescopes and of the Auxiliary Telescopes, as well as the construction of the infrastructure. Several measurement campaigns have confirmed that both opto-mechanical and environmental specifications are met.

The status of the VLTI sub-systems is as follows: 


\section{Test-Siderostats}

For tests with the VLTI and the instruments two Test-Siderostats were designed and built. The free aperture of $400 \mathrm{~mm}$ allows for about 20 stars to be observed in the N-band at $10 \mu \mathrm{m}$. Since for the VLTI Control System the Siderostats will 'look' the same as the UTs and ATs, it is planned to commission the VLTI with the Siderostats. They will be located on the AT stations and they will, thus, be able to use baselines between 8 and $200 \mathrm{~m}$. The systems are readily manufactured and they will be delivered to Paranal in January 2000. The implementation of the Siderostat control software will be finished by May 2000. It is planned to have first fringes with the Siderostats and VINCI in March 2001.

\section{Delay Line Systems}

Three Delay Lines have been ordered, the first two will be installed in the Delay Line Tunnel in July 2000, the third one in December 2000. The systems consist of the cat's-eye mirror system retroreflecting the incoming parallel beam, and of the carriage transporting the cat's-eye along the delay lines. The three mirror optical design of the cat's-eye has a variable curvature mirror in the focus of the cat's-eye in order to reimage the telescope pupil into a fixed position in the VLTI laboratory while the Delay Line System is tracking. The cat's-eye can handle two input beams as required for a dual feed system. A total of eight Delay Line Systems can be hosted in the Delay Line Tunnel.

\section{Auxiliary Telescope Systems}

In June 1998 two 1.8-m Auxiliary Telescopes were ordered, and in June 1999 the option for the third AT was exerted. The first two telescopes will be ready for the VLTI in June 2002, the third in October 2002. The telescopes are relocatable on 30 stations of the VISA (VLT Interferometer Sub-Array) providing baselines between 8 and $200 \mathrm{~m}$. Using three telescopes and, thus, three baselines at the same time will allow the application of closure phase techniques eliminating the influence of atmospheric turbulence on fringe position. Each AT will be equipped with a tip-tilt system correcting for the fast image motion induced by atmospheric turbulence. Under the seeing conditions at Paranal tiptilt correction on a 1.8-m telescope in the near infrared means almost diffraction limited image quality. One should note that the ATs are available exclusively for the VLTI, forming an observatory that is operated independently of the Unit Telescopes.

\section{Coudé Optical Trains and Transfer Optics}

The five mirrors after the tertiary mirror of the Unit Telescopes form the Coudé Optical Train with a field of view of 2 arcmin. The last mirror (M8) before the Coudé Focus is very close to the telescope exit pupil and can be replaced by the deformable mirror of the adaptive optics system. The image quality on axis at $2.2 \mu \mathrm{m}$ is diffraction limited on the optical axis and better than $97 \%$ over the full field of view. The Coudé Optical Trains for all four UTs have been ordered and will be installed at Paranal in October 2000.

The transfer optics between the Coudé Focus and the Delay Lines are currently being designed. The concept is to avoid the need of human intervention 
in the delay line tunnel when switching between AT stations or UTs. Thus, the mirrors in the Delay Line Tunnel reflecting the light into the Delay Lines and from the Delay Lines into the VLTI Laboratory will be controlled remotely.

\section{VLTI Laboratory}

The concept driver for the optical layout of the VLTI Laboratory was to provide the same beam diameter for the interferometric instruments both when observing with UTs or ATs. Therefore, a beam compressor reduces the $80 \mathrm{~mm}$ beam diameter from the UTs to $18 \mathrm{~mm}$ matching the diameter of $18 \mathrm{~mm}$ provided by the ATs. The nominal position of equal optical path length is set for all beams at the same distance after the switchyard to simplify the optical alignment of the interferometric instruments.

\section{Phase A - Two Telescope Interferometry}

The VLTI in its first phase will combine two telescopes, and near- and midinfrared instrumentation will be available. An adaptive optics system for the UTs and a fringe sensor unit (FSU) will be implemented as well. In the near infrared, adaptive optics on the UTs is mandatory if the effective telescope aperture is not to be reduced to the size of the Fried Parameter, $r_{0}$, which is about $1 \mathrm{~m}$ in the near infrared.

\section{VINCI - The Commissioning Instrument}

The commissioning instrument of the VLTI is a conceptual copy of FLUOR, the near-infrared interferometric instrument of the IOTA interferometer on Mount Hopkins in Arizona. The idea is to limit the technical risk and to facilitate the commissioning of the VLTI by using the design of an existing well functioning instrument. The main component of VINCI is a fiber beam combiner using the light from two telescopes as input and producing four outputs, two photometric and two interferometric signals. By varying the OPD between the beams with an internal modulator a temporally modulated fringe pattern is produced on the detector. In addition to serving as the interferometric instrument VINCI provides alignment tools and reference sources for the VLTI and the scientific instruments. VINCI will be delivered to Paranal in January 2001.

\section{MIDI}

An interferometric instrument in the mid-infrared at $10 \mu \mathrm{m}$ in combination with 8-m telescopes is a novelty, promising exciting results on new types of objects.

MIDI is being designed and built by a European consortium led by the MaxPlanck-Institute for Astronomy in Heidelberg. The design philosophy is to have a simple instrument concept combining two beams and providing a moderate spectral resolution $(\approx 200)$. The challenge lies in controlling the high thermal background. It is estimated that the individual beams will have an emissivity of about $50 \%$, which corresponds to an equivalent photon noise on the sky of 53 mJy per Airy disk (with $\lambda / \mathrm{D}=0.26$ " for an aperture of $8 \mathrm{~m}$ ) for a broad band $10 \mu \mathrm{m}$ filter $(\Delta \lambda=4 \mu \mathrm{m})$. Since the signal-to-noise-ratio scales as $\mathrm{S} / \mathrm{N} \propto \mathrm{D}^{2}$ the availability of the Unit Telescopes is a huge advantage over smaller apertures. 
MIDI will be delivered to Paranal in June 2001; first light with the Siderostats is planned for September 2001.

\section{AMBER}

The near infrared instrument of the VLTI, AMBER, will operate between 1 and $2.5 \mu \mathrm{m}$, at first with two telescopes with a spectral resolution up to 10000 . As noted above, for UT observations in the near-infrared adaptive optics is mandatory. The magnitude limit of AMBER on the UTs is expected to reach $\mathrm{K}=20$ when a bright reference star is available (i.e. with a dual feed facility) and $\mathrm{K}=$ 14 otherwise. The European consortium in charge of designing and manufacturing this instrument is led by the Universities of Nice and Grenoble. AMBER has been designed for three beams to enable imaging through phase closure techniques. It is planned to start commissioning AMBER with the Siderostats in February 2002.

\section{MACAO}

The adaptive optics system MACAO will have a 60-actuator bimorph mirror and a curvature wavefront sensor. The deformable mirror will replace one of the mirrors of the Coudé optical train, thus requiring no additional optical elements. As noted above, MACAO is essential for all near- infrared instrumentation to be used with the Unit Telescopes including the Fringe Sensor Unit operating in the H-band. This means that also a mid-infrared instrument like MIDI needs adaptive optics in order to improve the limiting magnitude by using a Fringe Tracker.

The first MACAO system will be installed on one of the Unit Telescopes in January 2003. It is planned to have MACAO ready for interferometric observations with two UTs in June 2003. Clones of the MACAO system will be used for future VLT instrumentation like SINFONI. MACAO is an in-house development.

\section{Phase B - Imaging}

The main drivers for the second phase of the VLTI are increasing the sensitivity of the interferometer and adding imaging modes. In addition, an astrometric mode will open the door for many thrilling scientific programmes.

Increasing the sensitivity of the VLTI calls for a dual feed facility. Then, the sensitivity is improved by using a bright guide star for fringe tracking - similar to the guide star in adaptive optics for wavefront sensing - in one of the two feeds allowing increasing the exposure time on the science object in the other feed up to 10-30 minutes depending on the position in the sky. With a high-precision laser metrology system used to determine the internal optical path length, a dual feed system also provides an imaging mode. The VLTI dual feed facility PRIMA is described in the following.

Imaging with an interferometer relies on filling efficiently the uv-plane with many different baseline vectors. Although this can be done by observing with two telescopes in many different positions using a dual feed system, it is more efficient to use three or more telescopes at the same time and eliminate the influence of atmospheric turbulence by applying phase closure techniques. Therefore, the 
third and fourth delay line as well as the third Auxiliary Telescope are included in this phase.

\section{PRIMA}

The Phase Referenced Imaging and Micro-arcsec Astrometry (PRIMA) facility is a dual feed system adding a faint object imaging and an astrometry mode to the VLTI. PRIMA enables simultaneous interferometric observations of two objects - each with a maximum size of 2 arcsec - that are separated by up to 1 arcmin, without requiring a large continuous field of view. One object will then be used as a reference star for fringe tracking whilst the other object will be the science target. As a detector for PRIMA either the two scientific instruments MIDI and AMBER can be used making use of the fringe stabilisation provided by PRIMA, or a dedicated PRIMA detector for high precision astrometry that will be designed in the next years.

PRIMA is the key to access: 1) Higher sensitivity, the limiting magnitude will be about $\mathrm{K}=20,2$ ) imaging of faint objects with high angular resolution (<10 milli arcsec), 3) high precision astrometry $(\approx 10 \mu$ arcsec over a 10 arcsec field).

The principle of operation relies on finding within the isoplanatic angle $(\approx 1$ arcmin $)$ of the science target a sufficiently bright $\operatorname{star}(H \approx 12)$ that can be used as a reference star for the stabilisation of the fringe motion induced by atmospheric turbulence. Controlling all optical path lengths of the reference star and of the science star inside the interferometer $\left(O P D_{i n t}\right)$ with a laser metrology system introduces the capability of imaging faint objects and of determining the precise angular separation between the two stars. The measurement has to be repeated for up to $30 \mathrm{~min}$ in order to average out the variations of the differential OPD caused by atmospheric turbulence ( $\left.O P D_{\text {turb }}\right)$.

PRIMA can be subdivided into the four sub-systems: Star Separator, Laser Metrology System, Differential Delay Lines and Fringe Sensor Unit. These subsystems contain a number of technological challenges, in particular in the area of laser metrology, that require careful analysis of the possible technical solutions. Therefore, a feasibility study for the four subsystems was performed by Dornier Satellite Systems, Friedrichshafen, and by ONERA, Paris, to find the best technical solutions and to obtain a thorough financial estimate for the manufacturing of the subsystems. The results of the study became available in July 1999 and a Call for Tender for the manufacturing of the system is planned early in 2001 .

Dual feed observations with PRIMA can start as soon as the star separator, the fringe sensor unit and the differential delay lines are ready. Then, a reference star can be used for fringe tracking while integrating on the fainter science object as described above. If the fringe pattern can be stabilised over 10-100 sec the expected limiting magnitudes are about $\mathrm{K} \approx 16$ and $\mathrm{N} \approx 8$.

Phase information required for imaging and astrometry becomes available if the laser metrology system is installed. An OPD measurement accuracy of $500 \mathrm{~nm} \mathrm{rms}$ over $10 \mathrm{~min}$ sets the limiting magnitudes to about $\mathrm{K} \approx 20$ and $\mathrm{N} \approx$ 11. The Strehl ratio in the reconstructed image can be as good as $30 \%$ in the $\mathrm{K}$-band and $80 \%$ in the $\mathrm{N}$-band depending on the uv coverage. Reaching the final goal of $5 \mathrm{~nm}$ rms over $30 \mathrm{~min}$ allows $10 \mu$ arcsec astrometry. PRIMA shall be operational by 2003. 


\section{First Fringes and beyond}

The major challenge in making the VLTI work is the complexity of the system. Not only are the individual sub-systems high-tech instruments but they are also scattered over an area up to $200 \mathrm{~m}$ in diameter. The quality of the control system is a key issue for the smooth operation of the complete system. From the beginning of 2000 onwards, every 6 months major new sub-systems and instruments arrive at Paranal and have to be integrated into the VLTI. Thus, the strategy for commissioning has to be defined very carefully.

For the first milestone of the VLTI programme, First Fringes with the Siderostats and VINCI, we have adopted the following approach: after installation and commissioning of the Siderostats, of the Delay Lines and of the Transfer Optics, the optical alignment of the full system will be done with a Technical CCD. Then, all degrees of freedom but one can be adjusted and the dynamic behaviour of the delay lines can be tested with a star observed with the siderostats. With the commissioning instrument VINCI the last remaining degree of freedom, the optical path difference, will be adjusted to produce interferometric fringes before the end of 2000. After first fringes, in the first of half of 2001 the VLTI will be optimised by using different AT stations for the Siderostats and by integrating the third Delay Line into the system. While this is taking place the Coudé Optical Trains in the UTs and the remaining transfer optics are being installed.

The next instruments to arrive at Paranal are MIDI in the middle and AMBER at the end of 2001. Both science instruments will be commissioned with the Siderostats. MIDI can be tested shortly after first fringes on the Siderostats with the UTs even before the adaptive optics system MACAO arrives. After installation and commissioning of MACAO with the VLTI and with VINCI in the second half of 2002 AMBER will be tested on the UTs.

Also in 2002, a total of three Auxiliary Telescopes will be commissioned, first individually then with the VLTI and VINCI. The science instruments will follow suit. The first sub-systems of PRIMA arrive as well in 2002. During the course of 2003 both dual feed and closure phase operation can be tested with the VLTI.

In order to operate the VLTI as an observatory, support astronomers, instrument operators and engineers are required for regular operations and for maintenance. Although the number of nights with two (or more) UTs is probably restricted to a small fraction of the total number of available nights in the first few years, first the siderostats and then the Auxiliary Telescopes are available every night for VLTI observations. Thus, the planning for the VLTI has to foresee a full-time coverage with technical and scientific personnel. One has to keep in mind that the VLTI is running in parallel to the VLT observatory with Unit Telescopes and requires the same level of technical support for telescopes and instruments.

The final goal of the VLTI is to produce images with a few milli arcseconds resolution. A simulated point spread function when observing for eight hours with all four Uts has a very impressive allbeit elongated PSF with a full width at half maximum (FWHM) of about $4 \times 8$ milli arcsec for a wavelength of $2.2 \mu \mathrm{m}$. Having this goal in mind one must not forget that for several years the results 
will be individual measurement points in the uv plane. However, this does not diminish the scientific content of these results.

\section{The future}

The scenario of VLTI observations described in the article will without any doubt provide a wealth of scientific discoveries but it still does not exploit the full capacity of the infrastructure at Paranal. The layout of the interferometric tunnel and of the VISA array allows combining more than three telescopes providing either an even better coverage of the uv-plane or the operation of different instruments at the same time.

Therefore, the four final Delay Line Systems and all remaining Auxiliary Telescopes for a total of 8 ATs shall be installed for VLTI operations. The completion of the final phase will allow VLTI observations with 28 simultaneous baselines. The planning also has to include new, the second generation, instrumentation allowing the combination of six to eight telescopes and, at the same time, providing a dual feed facility for each pair of telescopes.

Beyond the fully equipped VLTI there have to be kilometric arrays with $8+$ $\mathrm{m}$ telescopes in order to drive the angular resolution into the sub milli arcsec range and to improve the sensitivity well beyond $\mathrm{K}=20$. The VLTI will be the stepping stone towards these observatories. 